\title{
LA THÉORIE DE LA JUSTICE DE NANCY FRASER EN TANT QUE CONTRIBUTION À L'ANALYSE DES POLITIQUES D'ACTION POSITIVE DANS L'ENSEIGNEMENT SUPÉRIEUR BRÉSILIEN
}

\section{ARTICLE ORIGINAL}

GUIMARÃES, Matheus De Oliveira ${ }^{1}$

GUIMARÃES, Matheus De Oliveira. La théorie de la justice de Nancy Fraser en tant que contribution à l'analyse des politiques d'action positive dans l'enseignement supérieur brésilien. Revista Científica Multidisciplinar Núcleo do Conhecimento. 04 année, Ed. 11, vol. 01, pp. 22-38. novembre 2019. ISSN: 24480959, Lien d'accès: https://www.nucleodoconhecimento.com.br/education-fr/theoriede-la-justice

\section{RÉSUMÉ}

Cet article cherche à présenter la formulation de la justice sociale nommée par la philosophe politique américaine Nancy Fraser à travers laquelle elle interprète le scénario social aujourd'hui de la reconnaissance binomique-redistribution. Nous présenterons des arguments théoriques qui peuvent être d'une valeur intéressante pour l'analyse, l'interprétation et l'évaluation des politiques éducatives brésiliennes directement liées au thème des politiques d'action positive. De la présentation de la théorie Frasean sur la justice sociale et la reconnaissance, nous cherchons à relier cette pensée, d'une manière panoramique, avec les actions positives visant à

\footnotetext{
1 Étudiant au doctorat en droit à l'éducation et aux politiques éducatives du Programme des cycles supérieurs en éducation de l'Université pontificale catholique du Minas Gerais (PucMinas). Maîtrise en éducation, liée à la ligne de recherche Diversité, Inclusion et pratiques éducatives, par l'Université fédérale d'Ouro Preto (UFOP). II est titulaire d'un diplôme en droit (UFOP), philosophie (ICSH) et pédagogie (FGD), spécialisé en pratiques pédagogiques (UFOP) et en gestion des politiques publiques (UFOP).
} 
l'enseignement supérieur mis en œuvre au Brésil - en particulier à partir de la décennie de 1990. À cette fin, les principaux arguments de Nancy Fraser sont démontrés, pour lesquels la justice exige aujourd'hui à la fois la redistribution des biens sociaux et de la richesse et la reconnaissance culturelle-valorativo des différences. Enfin, la relation théorique entre les actions positives et la théorie frasean est établie.

Mots-clés: actions positives, enseignement supérieur, justice sociale, Nancy Fraser, race, redistribution-reconnaissance.

\section{INTRODUCTION}

La Constitution brésilienne de 1988, dans son article 206, article I, et la Loi brésilienne sur les lignes directrices et les bases de l'éducation de 1996, à l'article 3, point I, établissent que l'enseignement doit être prévu en respectant le principe de l'égalité des conditions. Conformément à ce précepte constitutionnel, des politiques éducatives ont commencé à être formulées et mises en œuvre dans le but de réduire les inégalités sociales qui ont prévalu dans l'histoire du Brésil, discriminant divers segments de la société.

Au cours des deux dernières décennies, en ce qui concerne le traitement du thème racial au Brésil, des changements significatifs se sont produits dans le pays qui ont produit d'intenses transformations dans la société. Dans ce contexte, les actions positives sont incluses, comprises comme des initiatives visant à promouvoir l'inclusion sociale et, en même temps, les aspects singuliers des groupes et des individus sont valorisés.

Le lien entre les discussions sur l'éducation, le droit et les sciences politiques avec les thèmes liés aux questions de redistribution et de reconnaissance - et plus particulièrement en ce qui concerne la race - est largement fondé sur son indiscutable comprendre et faire face aux inégalités dans le contexte social, tant dans les relations de pouvoir que dans les connaissances, inéluctablement interconnectés (APPLE; BALLE; GANDIN, 2013; NÉ SOUS LA GLAS; LADSON-BILLINGS, 2013; APPLE, 2017). Malgré la compréhension que beaucoup ont d'un prétendu caractère 
épiphénomène de ce qui finirait par concerner les discussions sur la classe et le capitalisme, il est essentiel de comprendre la force relativement autonome des questions raciales et l'importance de se concentrer sur les particularités de la vie quotidienne de ceux qui vivent de telles situations (APPLE, 2017).

En ce sens, avec la nécessité d'assurer (et permanence) l'accès (et la permanence) à l'enseignement supérieur à ceux qui ont négligé leurs chances d'éducation, les politiques publiques brésiliennes ont cherché une universalisation tardive de l'enseignement supérieur et, peut-être en dépit de l'intention, ils finissent en ligne avec la perte de la qualité de l'enseignement sans démocratiser efficacement l'accès. Bien qu'au cours des deux dernières décennies, les indicateurs éducatifs se soient améliorés pour tous les secteurs d'enseignement et dans tous les groupes sociaux, de graves inégalités raciales sont encore observées, en particulier dans les premiers stades (HEILBORN; ARA-JO; BARRETO, 2010).

Malgré le débat sur les politiques d'action positive qui s'est concentré, en particulier depuis la dernière décennie, fortement sur le système de quotas pour les universités publiques, les actions de l'État impliquent des actions qui vont au-delà des quotas avec des caractéristiques et portée différenciée.

Bien qu'il soit possible d'affirmer qu'au gouvernement Fernando Henrique Cardoso il y avait déjà des initiatives fédérales visant la population noire, l'analyse des documents de l'époque révèle que la stratégie discursive et la politique dudit gouvernement était de promouvoir la reconnaissance sans investissements dans l'aspect redistributif, bien que l'inégalité ait été la principale justification des politiques de valorisation de la population noire (LIMA, 2010).

Le début de l'administration de Lula en 2003 marque un changement dense dans les politiques avec une perspective raciale. Comme l'affirme Lima (2010), jusqu'alors, la relation de l'État avec le Mouvement noir était d'extériorité, constituant essentiellement des acteurs sociaux en tant que plaignants et avec peu d'insertion dans l'appareil gouvernemental. Dans le gouvernement de Lula, cette relation découle de 
l'incorporation, dans ses cadres, de représentants noirs (donnant une plus grande visibilité aux exigences du mouvement).

Le fait est que le thème de l'enseignement supérieur est certainement celui qui mobilise le débat le plus public sur les actions positives, en particulier en ce qui concerne la mise en œuvre de politiques de quotas dans les établissements publics d'enseignement supérieur et les analyses sur les étudiants contingents. Cependant, comme l'affirme Lima (2010), la réaction sociale marquée à la politique des quotas n'a pas eu la même répercussion lorsque le gouvernement a mis en œuvre un programme d'actions positives dans le système privé - responsable de plus de $80 \%$ des inscriptions dans l'enseignement supérieur brésilien .

Les principales politiques publiques de portée fédérale avec découpe raciale dans l'éducation sont organisées aujourd'hui à la fois dans le domaine de la reconnaissance culturelle et dans celui de la redistribution. Au Brésil, en plus de la politique de quotas infâme (souvent comprise à tort comme une synthèse d'actions positives), PROUNI (University For All Program), par laquelle le gouvernement fédéral offre des bourses (intégrale et pour les étudiants à faible revenu afin qu'ils puissent étudier dans des collèges privés; fies (Fonds de financement des étudiants), un programme du ministère de l'Éducation visant à financer l'enseignement de premier cycle des étudiants inscrits dans des établissements non libres; et le PBP (Permanence Scholarship Program), une aide financière (offerte par le gouvernement fédéral aux étudiants à faible revenu des universités publiques fédérales et aux étudiants ayant des bourses complètes de PROUNI) qui vise à minimiser les inégalités sociales et contribuer à la permanence et à l'obtention du diplôme des étudiants de premier cycle dans une situation de vulnérabilité socio-économique.

Parmi ces politiques, Prouni est, bien sûr, l'action positive la plus redistributive d'un plus grand impact dans l'histoire de l'enseignement supérieur dans le pays. Créé en 2004, sous Lula, par la mesure provisoire 213/2004, Prouni a été institutionnalisé en 2005 par la loi 11 096/2005. Ce programme vise à accorder des bourses d'études complètes et partielles aux étudiants à faible revenu dans le domaine du premier cycle et des cours séquentiels de formation spécifique dans des établissements privés 
d'enseignement supérieur. Des exonérations fiscales sont offertes aux institutions anuu-des au Programme.

Corroborant l'idée d'une démocratisation tronquée de l'accès à l'enseignement supérieur par des actions positives, Pereira prédit, se référant indirectement aux résultats de Prouni :

[...] le secteur privé au Brésil p[responde]our $88 \%$ de l'offre de postes vacants et pour $71 \%$ des inscriptions, ce qui en fait l'un des plus importants au mond[...]e. Avec l'aide du réseau privé, l'offre de postes vacants pour l'enseignement supérieur couvre aujourd'hui $86 \%$ des diplômés du secondaire. [Contudo][...]seulement $10 \%$ des jeunes âgés de dix-huit à vingt-quatre ans y assistent (2009, p. 45).

Pereira (2009) ajoute que les postes vacants inactifs dans le secteur privé de l'IO étaient, avant la mise en œuvre de Prouni, à $49,5 \%$ - tandis que dans les $\mathrm{IHe}$ fédérales, ce taux était de 0,9\% et, dans l'État, de 4,7\%.

Sur les politiques d'expansion de l'enseignement supérieur, Pereira informe:

Le scénario économique que nous vivons démontre que les effets de la démocratisation de l'éducation ne conduisent pas à la démocratisation sociale, mais qu'ils peuvent produire de nouvelles inégalités plus douloureuses. (2009, p. 46).

Et c'est exactement comme proposition pour l'étude de ce contexte complexe qui indique l'analyse des théorisations présentées par Nancy Fraser (2008, 2003, 2002, 2000, 1997). Les actions positives visant à démocratiser l'accès et la permanence des minorités dans l'enseignement supérieur seraient-elles efficaces pour la promotion de la transformation sociale ? D'un point de vue économique, seraient-ils les mêmes efficaces? Et quels sont vos impacts sur le domaine de la reconnaissance culturellevalonisme de votre public cible?

II n'est pas prévu, dans ce texte, de réponses concluantes à ces questions. Cependant, on croit que la compréhension des arguments frasean dans ce contexte est d'une valeur intéressante pour cette analyse. 


\section{POLITIQUES ECONOMIC-DISTRIBUTIVES $X$ CULTURAL}

\section{VALUATION POLICIES: NANCY FRASER'S THEORIZATIONS ON THE DILEMMA REDISTRIBUTION-RECOGNITION AS ${ }^{[2]}$ A SUBSIDY FOR THE INTERPRETATION OF AFFIRMATIVE ACTION POLICIES IN BRAZILIAN HIGHER EDUCATION}

Nancy Fraser $(2008,2003,2002,2000,1997)$ souligne que la lutte pour la reconnaissance devient, à pas de l'avant, la forme paradigmatique du conflit politique depuis la fin du siècle dernier. Selon Fraser (2008, 2003, 2002, 2000, 1997), les demandes de reconnaissance de la différence alimentent les luttes des groupes mobilisés sous différents drapeaux : nationalité, ethnicité, race, sexe et sexualité. Par conséquent, l'identité de groupe va au-delà, dans ces conflits (appelés postsocialistes), l'intérêt des classes lui-même comme principal moyen de mobilisation politique : " la dom[a]ination culturelle supplante l'exploitation comme injustice fondamentale. Et la reconnaissance culturelle prend la place de la redistribution socioéconomique comme remède à l'injustice et à l'objectif de la lutte politique » (FRASER, 2008, p.11, traduction de la mienne).

Cependant, ce n'est pas tout, car les luttes pour la reconnaissance se développent dans un monde d'inégalités matérielles exacerbées, en particulier dans les pays du Sud.

Dans ce contexte, dans ses théorisations, Fraser (2008, 2003, 2002, 2000, 1997) soulève quelques questions qui exposeront son débat sur les dilemmes de la justice :

Comment, alors, voir l'éclipse d'un imaginaire socialiste centré sur des termes tels que l'intérêt, l'exploration et la redistribution? Et que devonsnous faire avec l'émergence d'une nouvelle imagerie politique centrée sur les notions d'identité, de différence, de domination culturelle et de reconnaissance ? Ce lacet représente-t-il un manque de fausse conscience ? Ou, au lieu de cela, serait-ce un moyen de compenser l'aveuglement culturel d'un paradigme marxiste mis à déconsidérer par l'effondrement du communisme soviétique? (FRASER, 2008, p.11, traduction de la mine, auteur griffins). 
Catégoriquement, Fraser $(2008,2003,2002)$ affirme qu'aucun de ces postes ne serait approprié, les qualifiant de «trop complets et sans nuances ». Plutôt que de simplement appuyer ou rejeter ce qui serait une tonne simple dans la politique identitaire, il faut considérer qu'il y a un défi intellectuel et pratique à relever : celui de développer une théorie critique de la reconnaissance qui identifie et assume le ne défend que les versions de la politique culturelle de différence qui peuvent être combinées de façon cohérente à la politique d'égalité sociale (FRASER, 2008, p. 1112).

En formulant ce projet, Fraser (2008) affirme que la justice exige aujourd'hui à la fois la redistribution et la reconnaissance, en proposant d'examiner la relation entre les deux. Selon l'auteur, cela signifierait, en partie, réfléchir à la façon de conceptualiser la reconnaissance culturelle et l'égalité sociale d'une manière qui se maintient à la fois - plutôt que de s'anéantir (puisqu'il y a plusieurs conceptions concurrentes entre eux). En outre, cela signifierait également la théorisation des moyens par lesquels la privation économique et le manque de respect culturel s'entremêlent et se soutiennent mutuellement. En ce sens, en outre, pour l'auteur, la justice exige également que les dilemmes politiques qui se posent soient clarifiés lorsque nous essayons de combattre les deux injustices en même temps (FRASER, 2008, p. 12).

Fraser prétend, comme l'un de ses buts:

(...) deux problèmes politiques qui sont maintenant dissociés les uns des autres. Ce n'est qu'avec l'intégration entre la reconnaissance et la redistribution que nous parviendrons à un cadre conceptuel adapté aux exigences de notre époque (FRASER, 2008, p.12, traduction de la mienne).

Deux modes génériques de compréhension de l'injustice sont présentés par Fraser (2008, p. 13-18).

La première serait celle qui fait référence à l'injustice économique, qui, selon elle, est enracinée dans la structure économique et politique de la société (exploitation, marginalisation économique et privation) et dont la conceptualisation de sa nature a 
déjà été entreprise, par le biais de grand effort, par des théoriciens égalitaires (FRASER, 2008, p. 13-14).

La deuxième façon de comprendre l'injustice, selon Fraser (2008), est culturelle ou symbolique, aggravant l'injustice dans les modèles sociaux de représentation, d'interprétation et de communication - dont les exemples, selon l'auteur, incluent la domination culturelle (concernant la soumission à des modèles d'interprétation et de communication associés à une autre culture, sans rapport et/ou hostile à la sienne); dissimulation (relative à l'invisibilisation par l'effet des pratiques communicatives, interprétatives et de représentation consenties par la culture elle-même); et le manque de respect (caractérisé par la diffamation et la disqualification systématique dans les représentations culturelles publiques stéréotypées et/ou dans les interactions de la vie quotidienne) (FRASER, 2008, p. 14).

Cependant, malgré les distinctions entre ces deux formes d'injustice (socioéconomique et culturelle), Fraser $(2008,2003,2002,2000)$ affirme que les deux sont nettement présentes dans les sociétés contemporaines. Les deux sont enracinés dans des processus et des pratiques qui favorisent les désavantages pour certains groupes par rapport à d'autres. En ce sens, l'une et l'autre forme d'injustice doivent être corrigées, quoique de différentes manières.

Pour Fraser (2008, 2003, 2002, 2000), le remède à l'injustice économique serait une sorte de restructuration politico-économique qui pourrait impliquer la redistribution des revenus, la réorganisation de la division du travail, le contrôle démocratique des investissements ou transformation d'autres structures économiques de base. Le philosophe politique attribue à cet ensemble de remèdes à l'injustice économique le terme générique de redistribution.

En revanche, selon l'auteur, le remède à l'injustice culturelle serait une sorte de changement culturel ou symbolique. Cela pourrait impliquer la valorisation des identités irrespectueuses (ainsi que les produits culturels des groupes diffamés), l'appréciation positive de la diversité culturelle, et, plus radicalement, pourrait englober une transformation globale des normes sociales représentation, interprétation et 
communication, afin de transformer le sens de soi et de tous les hommes. Fraser $(2008,2003,2002,2000)$ donne à cet ensemble de recours à l'injustice culturelle le terme reconnaissance générique.

Pour l'auteur (FRASER, 2008), lorsqu'il s'agit de collectivités qui s'approchent du type idéal de la classe exploitée, on est confronté à des injustices distributives qui manquent de remèdes redistributifs (dont la logique serait de promouvoir l'homogénéisation et mal caractériser les groupes). Lorsque, à notre tour, nous traitons des collectivités qui approchent le type idéal de sexualité méprisée, par exemple, les injustices de discrimination négative sont confrontées qui ont besoin de remèdes de reconnaissance (dont la logique, à son tour, serait la valorisation de la groupe de la reconnaissance de leurs spécificités). Cependant, à mesure qu'il s'éloigne des extrémités, les choses s'estompent et la différenciation devient beaucoup plus complexe et délicate. Selon Fraser (2008), lorsqu'on considère les collectivités situées dans la région intermédiaire du spectre conceptuel, il existe des types hybrides qui mélangent les caractéristiques de la classe exploitée avec les caractéristiques de la sexualité méprisée. Ces collectivités sont appelées, comme le prédit l'auteur, bivalentes. Ils diffèrent en tant que communautés en raison de la structure économique politique et de la structure culturelle-valorisée de la société :

Ces collectivités bivalentes (opprimées ou subordonnées) subissent des injustices qui remontent simultanément à l'économie politique et à la culture. Bref, comme Fraser l'informe, ces collectivités peuvent souffrir d'une mauvaise répartition socioéconomique et d'un manquement culturel, de sorte qu'aucune de ces injustices n'est, en soi, un effet indirect de l'autre, mais à la fois primaire et co-original. Dans ce cas, ni les médicaments de redistribution ni ceux de reconnaissance ne seraient suffisants. Fraser déclare catégoriquement : "Les collectivités bivalentes manquent des d[redistribuição e reconhecimento]eux » (FRASER, 2008, p. 23, ma traduction).

Selon Fraser, le sexe et la race sont des paradigmes de collectivités bivalentes. Bien qu'elles aient des particularités non partagées entre elles, elles couvrent toutes deux les dimensions économiques et les dimensions culturelles-valoratives : «le genre et 
la race, par conséquent, impliquent à la fois la redistribution et la reconnaissance » (FRASER, 2008, p. 23, traduction de la mienne).

Ainsi, selon Fraser (2008, p. 25, ma traduction), « la race [...]est un mode bivalent de collectivité ».

D'une part, la lutte contre le racisme ressemble à la classe, étant un principe structurel de l'économie politique. À cet égard, la race structure la division capitaliste du travail. Les professions mal payées et à faible statut, celles ennuyeuses, sales et domestiques sont occupées de façon disproportionnée par les Noirs, [3]tandis que les professions les mieux rémunérées, avec un statut plus élevé, les cols blancs, les professionnels, les techniques et les sont principalement occupés par des Blancs.

En outre, selon l'auteur $(2008,2003,2002,2000,1997)$, la race structure également l'accès au marché du travail formel, constituant de vastes segments de la population noire en tant que sous-classe (non tenue même pour la tenue elle-même) et exclue du système Productif. En conséquence, il existe une structure politico-économique qui engendre des modes d'exploitation, de marginalisation et de privation marqués spécifiquement par la race : " cette structure constitue la race en tant que différenciation économique et politique caractéristiques » (2008, p. 26, ma traduction).

À cet égard, selon l'auteur (2008), l'injustice raciale apparaît comme une sorte d'injustice distributive qui appelle une indemnisation redistributive. Ainsi, d'une manière très similaire à la classe, la justice raciale exige la transformation de l'économie politique pour éliminer cette racialisation. Et la logique du remède est similaire à la logique liée à la classe : "il s'agit de faire la course hors des affaires » (FRASER, 2008, p. 26, ma traduction). Si la race n'est rien de plus qu'une différenciation économique et politique, la justice exige, comme le dit Fraser (2008, 2003, 2002, 2000), qu'elle soit abolie comme critère de différenciation, en ce sens.

Cependant, comme l'auteur l'a dit lorsqu'il s'agit de genre, la race, ainsi que cela, n'est pas seulement économique-politique. La race a également des dimensions culturelles- 
valoratives qui l'insèrent dans l'univers de la reconnaissance, englobant également des éléments plus semblables à la sexualité qu'avec la classe (FRASER, 2008, p. 26).

Comme le souligne Fraser (2008), un aspect basal du racisme est l'eurocentrisme caractérisé par la construction autorisée de normes qui favorisent les traits associés à la branquitude. À cela s'ajoute le racisme culturel, caractérisé à son tour par la disqualification généralisée des choses codées comme noires, brunes ou jaunes ( $p$. 26).

Comme dans le cas du genre, ces dommages-intérêts sont des injustices de reconnaissance et, par conséquent, la logique de la réparation est également d'accorder une reconnaissance positive à un groupe spécifiquement dévalué.

Fraser $(2008,2003,2002,2000,1997)$ désigne ainsi la race comme un mode bivalent de collectivité, avec un visage économique-politique et d'autres visages culturelsvaloratifs - les deux visages s'entrelacent pour se renforcer, dialectiquement - d'autant plus que le Les normes culturelles racistes et eurocentriques sont institutionnalisées dans l'État et dans l'économie, tandis que le désavantage économique subi par les Noirs restreint leur voix.

Pour compenser l'injustice raciale, l'économie politique et la culture sont déplacées. Mais, comme en témoigne l'auteur lorsqu'il s'agit de genre, le caractère bivalent de la race est aussi la source d'un dilemme.

Fraser $(2008,2003,2002,2000)$ affirme que le sexe et la race sont donc des modes de collectivité dilemmes. Contrairement à la classe, qui occupe une extrémité du spectre conceptuel présenté par l'auteur, et la sexualité, qui occupe l'autre pointe, le sexe et la race sont bivalents, étant impliqués simultanément dans la politique de redistribution et dans la politique de la Reconnaissance.

Mais comment dissoudre la différenciation en même temps et dissoudre la spécificité d'une collectivité méprisée ? 
Après avoir présenté le dilemme de la redistribution-reconnaissance d'une manière apparemment insoluble et de supposer que les remèdes redistributifs pour l'injustice économique et politique différencient toujours les groupes sociaux tandis que les remèdes de reconnaissance pour L'injustice culturelle-valonisant souligne toujours la différenciation du groupe social, Fraser (2008, p. 28) propose d'examiner les conceptions alternatives de la redistribution - d'une part - et la reconnaissance - d'autre part.

Par des recours affirmatifs pour l'injustice, Fraser comprend ceux qui visent à corriger les effets inégaux des arrangements sociaux sans la structure sous-jacente qui les engendre (2008, p. 28).

En revanche, par des remèdes transformateurs, l'auteur comprend ceux qui se concentrent sur la correction des effets inégaux en transformant la structure gerativa sous-jacente (2008, p. 28).

En ce qui concerne l'auteur, le point fondamental de contraste entre les recours affirmatifs et les remèdes transformateurs pour l'injustice est « les effets terminaux par rapport aux processus qui les produisent. Ce n'est pas un changement graduel par rapport à un changement apocalyptique » (FRASER, 2008, p. 28, ma traduction).

Fraser (2008) explique cette distinction, d'abord, dans le cas des recours pour l'injustice culturelle. Selon l'auteur, les remèdes affirmatifs pour de telles injustices sont associés à ce qu'elle appelle le «multiculturalisme dominant » - une sorte de multiculturalisme qui propose de compenser le manque de respect par la réévaluation des identités de groupe injustement dévalué (tout en laissant intact le contenu de ces identités et les différenciations de groupe qui les sous-tendent). En revanche, les remèdes transformateurs sont associés à la déconstruction, compensant le manque de respect par la transformation de la structure culturelle-valorative sous-jacente. Comme l'affirme Fraser, déstabilisant les identités de groupe existantes et les différenciations, ces recours augmenteraient non seulement l'estime de soi des membres de groupes qui ne manquent pas de respect, mais transformeraient aussi le sentiment d'appartenance et l'affiliation de tous (p. 28-29). 
Pour l'auteur, il ne s'agit pas de dissoudre toute différence dans une identité humaine unique et universelle, mais plutôt de maintenir un champ de différences multiples, pas binaires, fluides, toujours en mouvement :

Bien que les recours en reconnaissance positive tendent à promouvoir les différenciations de groupe existantes, les recours en reconnaissance transformatrice tendent à long terme à les déstabiliser afin de faire place à de futurs regroupements (FRASER, 2008, p. 30, ma traduction).

Des distinctions similaires s'appliquent aux recours en cas d'injustice économique. Comme le dit Fraser (2008, 2003, 2002), les recours positifs pour ce type d'injustice sont historiquement associés à l'État-providence libéral, cherchant, par leur intermédiaire, à indemniser les personnes en phase terminale de mauvaise répartition (tout en laissant la plus grande partie de la structure économique et politique sousjacente). Les remèdes transformateurs, contre, s'associent historiquement au socialisme, cherchant à compenser la distribution injuste par la transformation de la structure économique et politique existante.

Fraser affirme qu'une approche visant à compenser les injustices de distribution peut finir par créer des injustices de reconnaissance (2008, p. 31).

La redistribution positive suppose une conception universaliste de la reconnaissance (valeur morale égale des personnes; droit de Fraser $(2008,2003,2002)$ comme un engagement formel à la reconnaissance). Cependant, la pratique de la redistribution positive, si elle est prolongée, tend à mettre en branle une dynamique secondaire de reconnaissance stigmatisante qui contredit son propre engagement formel envers I'universalism ${ }^{[4]}$ e. L'auteur (2008), qui oppose cette logique à des remèdes transformateurs pour les injustices distributives de classe, prédit qu'ils combinent des programmes de protection sociale universalistes, des impôts élevés, des politiques macroéconomiques visant à créer le plein emploi, un vaste secteur public non marchand, des propriétés publiques et/ou collectives importantes, et des décisions démocratiques concernant les priorités socio-économiques fondamentales. Les recours transformateurs en matière de redistribution tendent à dissoudre la différenciation des classes, réduisant ainsi les inégalités sociales sans créer de 
classes stigmatisées de personnes vulnérables considérées comme bénéficiaires d'une générosité particulière. Fraser affirme que ces recours tendent à promouvoir la réciprocité et la solidarité dans les relations de reconnaissance, ce qui permet de remédier à la compensation des injustices de redistribution pour aider, aussi, à compenser certaines injustices de reconnaissance (2008, p.31-33)

Fraser (2008) affirme que, comme la redistribution positive, la redistribution transformatrice (en général) présuppose une conception universaliste de la reconnaissance : la valeur morale égale des personnes. Cependant, contrairement à la redistribution positive, la pratique de la redistribution transformatrice tend à ne pas dissoudre cette conception.

De cette discussion, après avoir examiné seulement les cas typiques-idées pures aux deux extrémités du spectre conceptuel, et de comparer les effets divergents des remèdes positifs et transformateurs à des injustices distributives enracinées économiquement - d'une part - et ceux de la reconnaissance culturellement enracinée - d'autre part - on voit que: 1. les remèdes affirmatifs tendent généralement à favoriser la différenciation de groupe, tandis que les remèdes transformateurs tendent à déstabiliser et à brouiller cette différenciation ; et 2. Les recours en redistribution positive peuvent engendrer une protestation contre le mépris, tandis que les recours de redistribution transformateurs peuvent aider à compenser certaines formes de nonreconnaissance. En ce sens, Fraser $(2008,2003,2002)$ propose un moyen de reformuler le dilemme de la redistribution-reconnaissance.

Toutefois, en concluant cette discussion, Fraser soulève la question suivante :

[...] en ce qui concerne les groupes soumis aux deux types d'injustice, quelle serait la combinaison de recours qui permettrait le mieux de minimiser (sinon d'éliminer pour de bon) les interférences mutuelles qui surviennent lorsqu'il s'agit de redistribuer et de reconnaître le même temps? (2008, p. 33, ma traduction).

Et cela devrait encore être problématique. 


\section{CONSIDÉRATIONS FINALES : LA CONTEXTUALISATION DU DÉBAT FRASEANO À LA RÉALITÉ DE L'ENSEIGNEMENT SUPÉRIEUR AU BRÉSIL}

Il est affirmé que le Brésil, parce qu'il est caractérisé par de nombreuses inégalités, de différents ordres, est marqué comme un modèle paradigmatique pour réfléchir à l'application de la théorie phraséane. En ce sens, les théorisations de Fraser sont proposées pour l'analyse du scénario éducatif brésilien.

Comme ferreira (2010) le prétend, la modernité, à son arrivée au Brésil, a trouvé un pays avec des marques de conservatisme et de traditionalisme - une situation qui, en quelque sorte, reste à ce jour dans divers secteurs sociaux. Au Brésil, les préjugés et l'exclusion raciale ont eu lieu (et conservent encore, dans une certaine mesure) d'une manière voilée, non institutionnalisée et ouvertement reconnue. Cela compromet, dans de nombreux cas, les processus d'action du gouvernement. Des actions telles que les politiques d'action positive sont considérées à plusieurs reprises comme un affront à l'égalité des chances - même si l'inégalité, la ségrégation et les préjugés sont visibles (FERREIRA, 2010).

C'est en ce sens que la performance des pouvoirs publics en vue de réduire les inégalités sociales peut être comprise à partir des théorisations de Fraser $(2008,2003$, 2002,2000 ) sur la reconnaissance et la redistribution. II est essentiel que les politiques de redistribution et de reconnaissance ne se contredisent pas et, en outre, soient guidées par le respect mutuel (APPLE, 2017, p. 32).

Les politiques d'action positive font partie de Fraser (2008, 2003, 2002), comme remède contre les injustices sociales. Toutefois, comme le prédit Ferreira (2012), un point important à prendre en considération dans cette discussion fait référence au fait que, toujours d'un point de vue frasean, les actions positives ne seraient pas des garanties absolues afin de modifier positivement la structure qui génère des inégalités. C'est parce que, peut-être, a priori, ils ne reconnaissent que la nécessité de la distribution à des groupes historiquement affaiblis, mais ne résolvent pas 
nécessairement les impasses structurelles qui génèrent de telles inégalités. On s'attend à ce que les bénéficiaires de ces politiques puissent, à grande échelle et à long terme, transmettre leurs résultats de croissance socio-économique et culturelle de manière intergénérationnelle afin que leurs descendants n'aient plus besoin de politiques. de ce type de rester à la hausse ou d'atteindre des niveaux de revenu et de statut similaires à ceux de la population qui ont historiquement reçu ces droits au Brésil.

\section{RÉFÉRENCES}

APPLE, Michael W. A educação pode mudar a sociedade?. Petrópolis: Vozes, 2017.

APPLE, Michael W.; BALL, Stephen J.; GANDIN, Luís Armando. Mapeamento da sociologia da educação: contexto social, poder e conhecimento. In: Sociologia da Educação: análise internacional. Michael W. Apple; Stephen J. Ball; Luís Armando Gandin (orgs.). Porto Alegre: Penso, 2013.

LIMA, Márcia. Ações afirmativas no governo Lula. In: Revista Novos Estudos. 2010.

FERREIRA, Wallace. Justiça e reconhecimento em Nancy Fraser. Teresina: Jus Navigandi, 2012.

FRASER, Nancy. From Redistribution to Recognition? Dilemmas of Justice in a "Postsocialist" Age. In. Adding Insult to Injury. Nancy Fraser debates her critics. Edinburgh: Verso, 2008.

Justice Interruptus: Critical Reflections on the "Postsocialist" Condition. London: Routledge, 1997.

Redistribuição ou reconhecimento? Classe e status na sociedade contemporânea. In: Interseções - Revista de Estudos Interdisciplinares. UERJ, ano 4, n.1, 2002.

Recognition without Ethics? Theory, Culture \& Society, v. 18, p. 21-42, 2001. 
Da redistribuição ao reconhecimento? Dilemas da justiça na era pós-socialista. In: Democracia hoje: novos desafios para a teoria democrática contemporânea. Jessé Souza (org.). Brasília: Editora Universidade de Brasília, 2000.

FRASER, N.; HONNETH, A. Redistribution or recognition?: a political philosophical exchange. London; New York: Verso, 2003.

GILLBORN, David; LADSON-BILLINGS, Gloria. Educação e Teoria Racial Crítica. In: Sociologia da Educação: análise internacional. Michael W. Apple; Stephen J. Ball; Luís Armando Gandin (orgs.). Porto Alegre: Penso, 2013.

HEILBORN, M. L.; ARAÚJO, L.; BARReto, A. (Orgs). Gestão de Políticas Públicas em Gênero e Raça: GPP-GeR, Módulo I. Rio de Janeiro: CESPE; Brasília: Secretaria de Políticas Públicas para as Mulheres. 2010.

PEREIRA, Elisabete Monteiro de Aguiar. A universidade da modernidade nos tempos atuais. In: Revista Avaliação, v. 14, n. 1, p. 29-52. Campinas, 2009.

ZONINSEIN, Jonas; FERES JÚNIOR, João. Ação Afirmativa e Desenvolvimento. In: FERES JÚNIOR, João \& ZONINSEIN, Jonas (Orgs.). Ação Afirmativa e Universidade: experiências nacionais comparadas. Brasília: Editora Universidade de Brasília, 2006.

2. Toma-se, aqui, como referência principal para a análise que se propõe, a seguinte obra: FRASER, Nancy. From Redistribution to Recognition? Dilemmas of Justice in a "Postsocialist" Age. In. Adding Insult to Injury. Nancy Fraser debates her critics. Edinburgh: Verso, 2008.

3. "People of color", nos termos de Fraser.

4. Nos termos de Fraser, essa dinâmica secundária (estigmatizante) pode ser entendida como o efeito de reconhecimento prático da redistribuição afirmativa (2008, p. 31-32).

Soumis : octobre 2019. 
Approuvé : novembre 2019. 